\title{
A REMARK ABOUT VISCOSITY SOLUTIONS OF HAMILTON-JACOBI EQUATIONS AT THE BOUNDARY
}

\author{
P. E. SOUGANIDIS ${ }^{1,2}$
}

\begin{abstract}
We consider viscosity solutions of first-order partial differential equations of Hamilton-Jacobi type in bounded domains. We give criteria which identify boundary points at which the equation is automatically satisfied in the viscosity sense, if it holds in the interior. These complement some recent results of M. G. Crandall and R. Newcomb [3].
\end{abstract}

Introduction. The theory of scalar nonlinear first-order partial differential equations has been substantially developed with the introduction by M. G. Crandall and P.-L. Lions $[2]$ of the correct class of generalized solutions. We continue by recalling their definition. Let $K$ be a subset of $\mathbf{R}^{N}$ and $F: K \times \mathbf{R} \times \mathbf{R}^{N} \rightarrow \mathbf{R}$ be continuous (i.e., $\left.F \in C\left(K \times \mathbf{R} \times \mathbf{R}^{N}\right)\right)$. A function $u \in C(K)$ is called a viscosity solution of $F(y, u, D u) \leq 0$ on $K$ if for each real-valued function $\phi$ which is continuously differentiable in a neighborhood of $K$ and each local maximum $z \in K$ of $u-\phi$ relative to $K$ one has

$$
F(z, u(z), D \phi(z)) \leq 0 .
$$

Here $D \phi=\left(\phi_{y_{1}}, \ldots, \phi_{y_{N}}\right)$ is the gradient of $\phi$. We will use the notation $C^{1}(K)$ to mean the set of functions which are defined and continuously differentiable in a neighborhood of $K$. Similarly, a viscosity solution of $F(y, u, D u) \geq 0$ in $K$ is a $u \in C(K)$ such that for every $\phi \in C^{1}(K)$ and local minimum $z \in K$ of $u-\phi$ relative to $K$ one has

$$
F(z, u(z), D \phi(z)) \geq 0 .
$$

A viscosity solution of $F=0$ on $K$ is a function which is a viscosity solution of both $F \leq 0$ and $F \geq 0$. We also call viscosity solutions of $F \leq 0(F \geq 0)$ viscosity subsolutions (respectively, supersolutions) of $F=0$. In view of this definition viscosity solutions do not have to be differentiable at any point. The set $K$ is also general. In what follows, however, we will be concerned with cases in which $K$ satisfies $\Omega \subset K \subset \bar{\Omega}$, where $\Omega$ is an open subset of $R^{N}, \bar{\Omega}$ is its closure and $\partial \Omega$ is its boundary.

The notion of viscosity solutions is important in view of the interaction between equations of Hamilton-Jacobi type, control theory and differential games. The first

Received by the editors November 26, 1984.

1980 Mathematics Subject Classification. Primary 35L40, 35L45, 35L50.

Key words and phrases. Hamilton-Jacobi equations, boundary value problems, viscosity solutions.

${ }^{1}$ This work was completed while visiting the Institute for Mathematics and its Applications, University of Minnesota, Minneapolis, MN 55455.

${ }^{2}$ Partially supported under NSF Grant No. DMS-8401725 and ONR Grant No. N00014-83-K0542 . 
uniqueness theorems using this notion are proved in M. G. Crandall and P.-L. Lions [2]. M. G. Crandall, L. C. Evans and P.-L. Lions [1] provide a simpler introduction to the subject while the book by P.-L. Lions [6] and the review paper by M. G. Crandall and P. E. Souganidis [4] provide a view of the scope of the theory and references to much of the recent literature.

Our current interest is generated by some recent results of M. G. Crandall and R. Newcomb [3]. In particular, they gave criteria which identify boundary points at which an inequality is automatically satisfied in the viscosity sense if it holds in the interior of a set. Our main results, which we introduce and prove in $\S 1$, are of similar nature. The assumptions on $F$ are more restrictive here than in [3]; the results, however, apply to more general sets $K$ than [3]. Examples establishing the above remarks are also given.

1. Let $\Omega$ be an open subset of $\mathbf{R}^{N}$ and $\partial \Omega$ its boundary. We consider sub- and supersolutions $u \in C(\bar{\Omega})$ of an equation $F=0$ on $\Omega$. We want to identify a subset $I_{F}$ of $\partial \Omega$ (which following [3] we call the part of $\partial \Omega$ irrelevant for $F$ ) such that $u$ is a sub- or supersolution of $F=0$ on $\Omega \cup I_{F}$. Our concern is about the generality of $u, \Omega$ and $F$ under which such a result holds. We begin with a general open set $\Omega$. We need to define an appropriate set of normal directions at a point $z \in \partial \Omega$. We will do this using the properties of the function

$$
d(y)=\inf \left\{|y-w|^{2}: w \in \partial \Omega\right\}
$$

which is the square of the distance from $y$ to $\partial \Omega$. Associated with $d(y)$ is the set

$$
P y=\left\{w \in \partial \Omega: d(y)=|y-w|^{2}\right\}
$$

which consists of all points of $\partial \Omega$ which are nearest $y$. To describe the properties of $d$, we need the following definition.

DEFinition 1. For a function $f \in C(\Omega)$ and $x \in \Omega$,

$$
D^{+} f(x)=\left\{p \in \mathbf{R}^{N}: \varlimsup_{y \rightarrow x} \frac{f(y)-f(x)-p \cdot(y-x)}{|y-x|} \leq 0\right\} .
$$

We have then

Proposition 1. For every $x \in \Omega, D^{+} d(x) \neq \varnothing$. Moreover, if $P x$ is singleton,

$$
\operatorname{Dd}(x)=2(x-P x),
$$

where here $P x$ denotes the closest point.

We continue formulating the main results and give the proof of the proposition at the end of the section. The open ball of radius $r$ centered at $z \in \mathbf{R}^{N}$ will be denoted by $B_{r}(z)$, i.e., $B_{r}(z)=\left\{y \in \mathbf{R}^{N}:|y-z|<r\right\}$.

DEFINITION 2. Let $z \in \partial \Omega$. Then $z \in I_{F}$ if there is an $r>0$ such that for all $y \in \Omega \cap B_{r}(z)$ there exists $w \in P y$ such that for all $(u, p) \in \mathbf{R} \times \mathbf{R}^{N}$, all $\lambda>0$, and all $\nu \in D^{+} d(y)$,

$$
F(w, u, p+\lambda \nu) \leq F(w, u, p) .
$$

THEOREM 1. Let $F \in C\left(\bar{\Omega} \times \mathbf{R} \times \mathbf{R}^{N}\right)$ and $z \in I_{F}$. Let $u \in C\left(\Omega \cup I_{F}\right)$ be a Lipschitz continuous near $z$ viscosity solution of $F \leq 0(F \geq 0)$ on $\Omega$. Then $u$ is 
also a viscosity solution of $F \leq 0$ (respectively, $F \geq 0$ ) on $\Omega \cup\{z\}$. In particular, if $u$ is a viscosity solution of $F=0$ on $\Omega$, then it is a viscosity solution on $\Omega \cup\{z\}$.

Before proving this result, we want to discuss its relation with Theorem 1 of [3]. To begin with, Definition 2 imposes on $F$ stronger monotonicity assumptions than Definition 2 of $[3]$ as can be easily seen using Example 2 of $[3]$. In this case $(0,0)$ is not in $I_{F}$. On the other hand, however, Theorem 1 of [3] applies only to points $z \in \partial \Omega$ such that $P y$ is singleton for every $y$ in a neighborhood of $z$. Such an assumption cannot hold if, for example, $\partial \Omega$ has a corner at $z$. Finally, if $\Omega$ is of $C^{2}$ class the two theorems coincide. To illustrate the above remarks, we consider the following example.

EXAMPLE. Let $N=2$ and $(x, y)$ denote points of $\mathbf{R}^{2}$. Put $\Omega=\left\{(x, y) \in \mathbf{R}^{2}: 0<\right.$ $x, 0<y\}$ and

$$
F\left(x, y, u, u_{x}, u_{y}\right)=u-u_{x}-u_{y} .
$$

We have

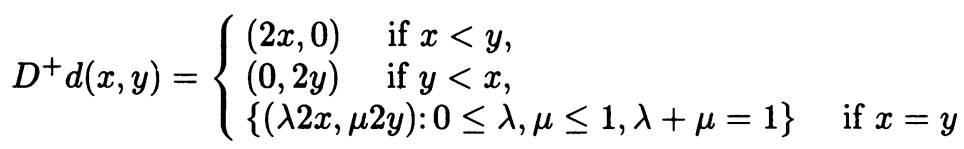

and it is straightforward to check that $(0,0) \in I_{F}$ and that it does not satisfy the assumptions of Theorem 1 of $[\mathbf{3}]$. In particular, every $(x, x)$ is not regular. $u \equiv 0$ is, however, a viscosity solution of $F=0$, which is also a viscosity solution of $F=0$ on $\Omega \cup\{(0,0)\}$.

PROOF OF THEOREM 1. Let $u \in C\left(\Omega \cup I_{F}\right)$ be a (locally) Lipschitz continuous viscosity solution of $F \leq 0$ in $\Omega$. Let $z \in I_{F}$ and put $\Omega_{z}=\Omega \cup\{z\}$. If $\phi \in C^{1}\left(\Omega_{z}\right)$ and $u-\phi$ has $z$ as a local maximum relative to $\Omega_{z}$, we want to prove that

$$
F(z, u(z), D \phi(z)) \leq 0 \text {. }
$$

Let $\varepsilon>0$. Using the arguments of the proof of Theorem 1 of $[\mathbf{3}]$, we obtain that, for $r$ sufficiently small,

$$
\Psi_{\varepsilon}(y)=u(y)-\phi(y)-\varepsilon / d(y)
$$

has a strict local maximum $y_{\varepsilon} \in \Omega \cap B_{r}(z)$ relative to $\Omega$ satisfying

$$
\left\{\begin{array}{ll}
\text { (i) } & y_{\varepsilon} \rightarrow z, \\
\text { (ii) } & \Psi_{\varepsilon}\left(y_{\varepsilon}\right) \rightarrow u(z)-\phi(z)
\end{array} \text { as } \varepsilon \rightarrow 0 .\right.
$$

The problem, however, is that we do not know whether $\operatorname{Dd}\left(y_{\varepsilon}\right)$ exists. To circumvent this difficulty we argue as follows.

For each $\varepsilon>0$ let $\zeta_{\varepsilon}$ be a smooth function, compactly supported in $0=\Omega \cap B_{r}(z)$ with the properties

$$
0 \leq \varsigma \leq 1 \text { and } \zeta\left(y_{\varepsilon}\right)=1 .
$$

For $\delta, \theta>0$ we define $\Phi_{\varepsilon}: 0 \times 0 \rightarrow \mathbf{R}$ by

$$
\Phi_{\varepsilon}(x, y)=u(x)-\phi(y)-\varepsilon / d(y)-2 M|x-y|^{2} / \theta^{2}+\delta \zeta(y)
$$


where $M=\sup \{|u(x)|: x \in \overline{0}\}$. We claim that for $\theta$ sufficiently small there exists $\left(x_{0}, y_{0}\right) \in \mathcal{O} \times \mathcal{O}$ such that

$$
\Phi\left(x_{0}, y_{0}\right)=\max _{0 \times 0} \Phi(x, y)
$$

Indeed

$$
\Phi(x, y) \leq \omega_{u}(\theta)+u(y)-\phi(y)-\varepsilon / d(y)+\delta \zeta_{\varepsilon}(y) \quad \text { for } x, y \in 0,
$$

where $\omega_{u}(\cdot)$ denotes the modulus of continuity of $u$. The definition of $y_{\varepsilon}$ and the previous inequality imply

$$
\Phi(x, y) \leq \begin{cases}\Phi\left(y_{\varepsilon}, y_{\varepsilon}\right)+\omega_{u}(\theta)-\delta & \text { for }(x, y) \in O \times\left(O \backslash \operatorname{supp} \zeta_{\varepsilon}\right) \\ \Phi\left(y_{\varepsilon}, y_{\varepsilon}\right)+\omega_{u}(\theta) & \text { for }(x, y) \in O \times \operatorname{supp} \zeta_{\varepsilon}\end{cases}
$$

Let $\left(x_{n}, y_{n}\right)$ be a maximizing sequence. If $\omega_{u}(\theta)<\delta$, it follows that for $n$ sufficiently large $y_{n} \in \operatorname{supp}_{\zeta_{\varepsilon}}$. Moreover, if $\left|x_{n}-y_{n}\right|>\theta$, then $\Phi\left(x_{n}, y_{n}\right)<\Phi\left(y_{n}, y_{n}\right)$. This finishes the proof of the claim provided $\theta$ is sufficiently small. It also implies that $\left|x_{0}-y_{0}\right| \leq \theta$.

Next observe that $x_{0}$ is a local maximum of $x \rightarrow u(x)-2 M\left|x-y_{0}\right|^{2} / \theta^{2}$. Therefore

$$
F\left(x_{0}, u\left(x_{0}\right), 4 M\left(x_{0}-y_{0}\right) / \theta^{2}\right) \leq 0 .
$$

Also $y_{0}$ is a local maximum of $y \rightarrow-\phi(y)-\varepsilon / d(y)-2 M\left(x_{0}-y\right)^{2} / \theta^{2}+\delta \zeta_{\varepsilon}(y)$. After a simple calculation which we leave to the reader, this yields

$$
(1 / \varepsilon) d^{2}\left(y_{0}\right)\left(D \phi\left(y_{0}\right)-4 M\left(x_{0}-y_{0}\right) / \theta^{2}-\delta D_{\zeta \varepsilon}\left(y_{0}\right)\right) \in D^{+} d\left(x_{0}\right),
$$

i.e.,

$$
4 M\left(x_{0}-y_{0}\right) / \theta^{2}=-\varepsilon p / d^{2}\left(y_{0}\right)+D \phi\left(y_{0}\right)-\delta D_{\zeta_{\varepsilon}}\left(y_{0}\right) \quad \text { for some } p \in D^{+} d\left(y_{0}\right) .
$$

Combining the above, we obtain

$$
F\left(x_{0}, u\left(x_{0}\right), D \phi\left(y_{0}\right)-\delta D_{\zeta_{\varepsilon}}\left(y_{0}\right)-\varepsilon p / d^{2}\left(y_{0}\right)\right) \leq 0 .
$$

Next let $z_{0} \in P y_{0}$ as in Definition 2 and write (1.11) as

$$
\begin{aligned}
& F\left(x_{0}, u\left(x_{0}\right), D \phi\left(y_{0}\right)-\delta D_{\zeta_{\varepsilon}}\left(y_{0}\right)-\frac{\varepsilon}{d^{2}\left(y_{0}\right)} p\right) \\
& \quad-F\left(y_{0}, u\left(x_{0}\right), D \phi\left(y_{0}\right)-\delta D_{\zeta_{\varepsilon}}\left(y_{0}\right)-\frac{\varepsilon}{d^{2}\left(y_{0}\right)} p\right) \\
& +F\left(y_{0}, u\left(x_{0}\right), D \phi\left(y_{0}\right)-\delta D_{\zeta_{\varepsilon}}\left(y_{0}\right)-\frac{\varepsilon}{d^{2}\left(y_{0}\right)} p\right) \\
& \quad-F\left(z_{0}, u\left(x_{0}\right), D \phi\left(y_{0}\right)-\delta D_{\zeta_{\varepsilon}}\left(y_{0}\right)-\frac{\varepsilon}{d^{2}\left(y_{0}\right)} p\right) \\
& +F\left(z_{0}, u\left(x_{0}\right), D \phi\left(y_{0}\right)-\delta D_{\zeta_{\varepsilon}}\left(y_{0}\right)-\frac{\varepsilon}{d^{2}\left(y_{0}\right)} p\right) \leq 0 .
\end{aligned}
$$

We treat the various terms of this inequality. First, since $u$ is Lipschitz continuous,

$$
\left|D \phi\left(y_{0}\right)-\delta D_{\zeta_{\varepsilon}}\left(y_{0}\right)-\varepsilon p / d^{2}\left(y_{0}\right)\right| \leq L,
$$

where $L$ is a Lipschitz constant for $u$ in $O$ (see [2, Lemma II.3]). Since $\left|x_{0}-y_{0}\right| \leq \theta$, we may use (1.13) and the continuity of $F$ to conclude that the difference comprised 
by the first two terms in (1.12) tends to 0 as $\theta \rightarrow 0$ for every $\delta$ and $\varepsilon$. As regards the last term, our assumptions imply that

$$
F\left(z_{0}, u\left(x_{0}\right), D \phi\left(y_{0}\right)-\delta D_{\zeta_{\varepsilon}}\left(y_{0}\right)\right) \leq F\left(z_{0}, u\left(x_{0}\right), D \phi\left(y_{0}\right)-\delta D_{\zeta_{\varepsilon}}\left(y_{0}\right)-\varepsilon p / d^{2}\left(y_{0}\right)\right) .
$$

To conclude we claim that

$$
\lim _{\varepsilon \rightarrow 0} \lim _{\delta \rightarrow 0} \lim _{\theta \rightarrow 0} y_{0}=\lim _{\varepsilon \rightarrow 0} \lim _{\delta \rightarrow 0} \lim _{\theta \rightarrow 0} z_{0}=z .
$$

Indeed, since $\left|x_{0}-y_{0}\right| \leq \theta$ and $y_{0} \in \operatorname{supp}_{\varsigma_{\varepsilon}}$,

$$
\lim _{\theta \rightarrow 0} y_{0}=\lim _{\theta \rightarrow 0} x_{0}=y_{1}(\varepsilon, \delta) \in \overline{\operatorname{supp} \zeta_{\varepsilon}}
$$

along any subsequence $\theta \rightarrow 0$. The fact that $\left(x_{0}, y_{0}\right)$ is a maximum of $\Phi$ yields

$$
u\left(x_{0}\right)-\phi\left(y_{0}\right)-\varepsilon / d\left(y_{0}\right)+\delta_{\zeta_{\varepsilon}}\left(y_{0}\right) \geq u(x)-\phi(x)-\varepsilon / d(x)+\delta_{\zeta_{\varepsilon}}(x) .
$$

Letting $\theta \rightarrow 0$ we obtain

$$
u\left(y_{1}\right)-\phi\left(y_{1}\right)-\varepsilon / d\left(y_{1}\right)+\delta \zeta\left(y_{1}\right) \geq u(x)-\phi(x)-\varepsilon / d(x)+\delta \zeta(x) .
$$

Next observe that along subsequences

$$
\lim _{\delta \rightarrow 0} y_{1}(\varepsilon, \delta)=y_{2}(\varepsilon) \in 0
$$

and

$$
u\left(y_{2}\right)-\phi\left(y_{2}\right)-\varepsilon / d\left(y_{2}\right) \geq u(x)-\phi(x)-\varepsilon / d(x) .
$$

Since $y_{\varepsilon}$ is a strict local maximum of $\Psi_{\varepsilon}$, we have $y_{2}=y_{\varepsilon}$. The second part follows from the above and the fact that $\left|y_{0}-z_{0}\right|^{2}=d\left(y_{0}\right)$. Combining all the previous observations we get (1.6), since the difference of the two middle terms in (1.12), in view of (1.13), (1.15) and the continuity of $F$, also tends to 0 as $\theta \rightarrow 0, \delta \rightarrow 0$, $\varepsilon \rightarrow 0$.

We continue by formulating another result similar to Theorem 2 of [3]. Here we will assume less on $u$ at the expense, however, of $F$.

DEFInITION 3. Let $z \in I_{F}$. Then $z$ is regular for $F$ if there exists an $r>0$ such that for all sequences $y_{n} \in B_{r}(z) \cap \Omega$ convergent to $z$, if $w_{n} \in P_{y_{n}}$ is given by Definition 1 , then for all $\lambda_{n}>0$ and $q_{n} \in \mathbf{R}^{N}$ satisfying

$$
\lambda_{n}\left|y_{n}-z_{n}\right|\left|q_{n}\right| \rightarrow 0
$$

we have

$$
\liminf _{n \rightarrow \infty} F\left(y_{n}, u, p-\lambda_{n} q_{n}\right)-F\left(w_{n}, u, p-\lambda_{n} q_{n}\right) \geq 0
$$

uniformly for bounded $u$ and $p$.

We have

THEOREM 2. Let $F \in C\left(\bar{\Omega} \times \mathbf{R} \times \mathbf{R}^{N}\right)$. Let $z \in I_{F}$ be regular for $F$ and $u \in C\left(\Omega \cup I_{F}\right)$ be a viscosity solution of $F \leq 0(F \geq 0)$ on $\Omega$. Then $u$ is also a viscosity solution of $F \leq 0$ (respectively, $F \geq 0$ ) on $\Omega \cup\{z\}$. In particular, if $u$ is a viscosity solution of $F=0$ on $\Omega$, then it is a viscosity solution on $\Omega \cup\{z\}$.

PROOF. The proof follows the proof of Theorem 1 exactly up to the discussion of the four middle terms of (1.12). To continue we make the following observation concerning $D^{+} d\left(y_{0}\right)$ for $y_{0} \in \Omega$.

$$
\text { If } p \in D^{+} d\left(y_{0}\right) \text {, then } p / 2 d^{1 / 2}\left(y_{0}\right) \in D^{+} d^{1 / 2}\left(y_{0}\right) \text {. }
$$


Indeed, Definition 1 and (1.1) imply

and thus (1.18).

$$
d^{1 / 2}(y)-d^{1 / 2}\left(y_{0}\right) \leq \frac{p}{d^{1 / 2}(y)+d^{1 / 2}\left(y_{0}\right)} \cdot\left(y-y_{0}\right)+\frac{o\left(\left|y-y_{0}\right|\right)}{d^{1 / 2}(y)+d^{1 / 2}\left(y_{0}\right)}
$$

Next observe that in view of (1.18) and the fact that $d^{1 / 2}$ is Lipschitz continuous with constant $1,(1.13)$ still holds with a constant $L$ independent of $\theta$. Thus the difference of the first two terms again goes to 0 .

Next observe that (1.15) still holds and, in particular,

$$
\lim _{\delta \rightarrow 0} \lim _{\theta \rightarrow 0} y_{0}=y_{\varepsilon} .
$$

This implies that (1.13) still holds with a constant which is independent of $\theta$ but may depend on $\varepsilon$. Thus the difference of the first two terms again goes to 0 . After sending $\theta \rightarrow 0$, we are left with the difference

$$
\begin{aligned}
& F\left(y_{1}, u\left(y_{1}\right), D \phi\left(y_{1}\right)-\delta D_{\zeta_{\varepsilon}}\left(y_{1}\right)-\frac{\varepsilon}{d^{2}\left(y_{1}\right)} q_{1}\right) \\
& -F\left(z_{1}, u\left(y_{1}\right), D \phi\left(y_{1}\right)-\delta D_{\zeta_{\varepsilon}}\left(y_{1}\right)-\frac{\varepsilon}{d^{2}\left(y_{1}\right)} q_{1}\right)
\end{aligned}
$$

with $\left|q_{1}\right| \leq 2 d^{1 / 2}\left(y_{1}\right)$. Sending $\delta \rightarrow 0$ we obtain the difference

$$
F\left(y_{2}, u\left(y_{2}\right), D \phi\left(y_{2}\right)-\frac{\varepsilon}{d^{2}\left(y_{2}\right)} g_{2}\right)-F\left(z_{2}, u\left(y_{2}\right), D \phi\left(y_{2}\right)-\frac{\varepsilon}{d^{2}\left(y_{2}\right)} q_{2}\right),
$$

with $\left|q_{2}\right| \leq 2 d^{1 / 2}\left(y_{2}\right)$. The latter follows from (1.18) and the fact that $d^{1 / 2}$ is Lipschitz continuous with constant 1 . However, $D \phi\left(y_{2}\right)$ and $u\left(y_{2}\right)$ are bounded and

$$
\left|y_{2}-z_{2}\right| \frac{\varepsilon}{d^{2}\left(y_{2}\right)}\left|q_{2}\right| \leq \frac{2 \varepsilon}{d\left(y_{2}\right)} \rightarrow 0
$$

as follows immediately from (1.8) and the definition of $\Psi_{\varepsilon}$.

Applying (1.17) we complete the proof.

REMARK 1. Some of the arguments of the proof of Theorem 1 are similar to the ones used by L. C. Evans [5] in proving the single-valuedness of the Hamilton-Jacobi operator.

REMARK 2. One can formulate uniqueness theorems corresponding to Theorems 1 and 2. There are many possible variants of these results, which resemble the ones of $[\mathbf{3}]$. Thus we refer the interested reader to $[\mathbf{3}]$.

REMARK 3. In view of the above results as well as the results of [3] one can formulate very general theorems concerning domain of dependence.

We conclude the section with few remarks about the proof of Proposition 1. The second part is standard and we leave it for the reader.

To prove that $D^{+} d\left(y_{0}\right) \neq \varnothing$ for every $y_{0}$ we argue as follows. For $y$ near $y_{0}$ let $w_{y}$ and $w_{0}$ be minimizers of $(1.1)$ on $K$. Then

$$
\left|y-w_{y}\right|^{2}-\left|y_{0}-w_{y}\right|^{2} \leq d(y)-d\left(y_{0}\right) \leq\left|y-w_{0}\right|^{2}-\left|y_{0}-w_{0}\right|^{2}
$$

Subtract $2\left(y_{1}-y_{0}\right)\left(y_{0}-w_{0}\right)$ from the middle and right-hand term of (1.19) and divide by $\left|y-y_{0}\right|$. This gives

and thus the result.

$$
\limsup _{y \rightarrow y_{0}} \frac{d(y)-d\left(y_{0}\right)-2\left(y_{0}-w_{0}\right)\left(y-y_{0}\right)}{\left|y-y_{0}\right|} \leq 0
$$




\section{REFERENCES}

1. M. G. Crandall, L. C. Evans and P.-L. Lions, Some properties of viscosity solutions of Hamilton-Jacobi equations, Trans. Amer. Math. Soc. 282 (1984), 487-502.

2. M. G. Crandall and P.-L. Lions, Viscosity solutions of Hamilton-Jacobi equations, Trans. Amer. Math. Soc. 277 (1983), 1-42.

3. M. G. Crandall and R. Newcomb, Viscosity solutions of Hamilton-Jacobi equations at the boundary, Proc. Amer. Math. Soc. 94 (1985), 283-290.

4. M. G. Crandall and P. E. Souganidis, Developments in the theory of nonlinear first-order partial differential equations (Proc. Internat. Sympos. on Differential Equations, Birmingham, Alabama, 1983, I. W. Knowles and R. T. Lewis, eds.), North-Holland, Amsterdam, 1984.

5. L. C. Evans, Some max-min methods for the Hamilton-Jacobi equation, Indiana Univ. Math. J. 33 (1984), 31-50.

6. P.-L. Lions, Generalized solutions of Hamilton-Jacobi equations, Pitman, Boston, Mass., 1982.

Lefschetz Center for Dynamical Systems, Division of ApPlied MathematICS, BROWN UNIVERSITY, PROVIDENCE, RHODE ISLAND 02912 